SLAC-PUB-10716

\title{
PLANCK-SIZE BLACK HOLE REMNANTS AS DARK MATTER
}

\author{
PISIN CHEN \\ Stanford Linear Accelerator Center \\ Stanford University, Stanford, CA 94309, USA \\ chen@slac.stanford.edu
}

\begin{abstract}
While there exist various candidates, the nature of dark matter remains unresolved. Recently it was argued that the generalized uncertainty principle (GUP) may prevent a black hole from evaporating completely, and as a result there should exist a Plancksize black hole remnant (BHR) at the end of its evaporation. If a sufficient amount of small black holes can be produced in the early universe, then the resultant BHRs can be an interesting candidate for DM. We demonstrate that this is indeed the case for the hybrid inflation model. By assuming BHR as DM, our notion imposes a constraint on the hybrid inflation potential. We show that such a constraint is not so fine-tuned. Possible observational signatures are briefly discussed.
\end{abstract}

\section{Introduction}

It is by now widely accepted that dark matter (DM) constitutes a substantial fraction of the present critical energy density in the universe. However, the nature of DM remains an open problem. There exist many DM candidates, most of them are nonbaryonic weakly interacting massive particles (WIMPs), or WIMP-like particles. ${ }^{1}$ Figure 1 shows the masses and cross sections of WIMP (or WIMP-like) candidates. ${ }^{2}$ By far the DM candidates that have been more intensively studied are the lightest supersymmetric (SUSY) particles such as neutralinos or gravitinos, and the axions (as well as the axinos). There are additional particle physics inspired DM candidates. ${ }^{1}$ A candidate which is not as closely related to particle physics is the relics of primordial black holes (PBHs) ${ }^{3,4}$ Earlier it was suggested that PBHs are a natural candidate for DM. ${ }^{5}$ More recent studies ${ }^{6}$ based on the $\mathrm{PBH}$ production from the "blue spectrum" of inflation demand that the spectral index $n_{s} \sim 1.3$, but this possibility may be ruled out by recent WMAP experiment. ${ }^{7}$ There are also other studies on the idea of PBH as DM. ${ }^{8}$

One weakness of the notion of PBH as DM is the ambiguity on the final property of small black holes. The standard view of black hole thermodynamics ${ }^{9,10}$ does not provide an answer as to whether a small black hole should evaporate entirely, or leave something else behind, which we refer to as a black hole remnant (BHR). Numerous calculations of black hole radiation properties have been made from different points of view, ${ }^{11}$ and some hint at the existence of remnants, but none appear to give a definitive answer. 


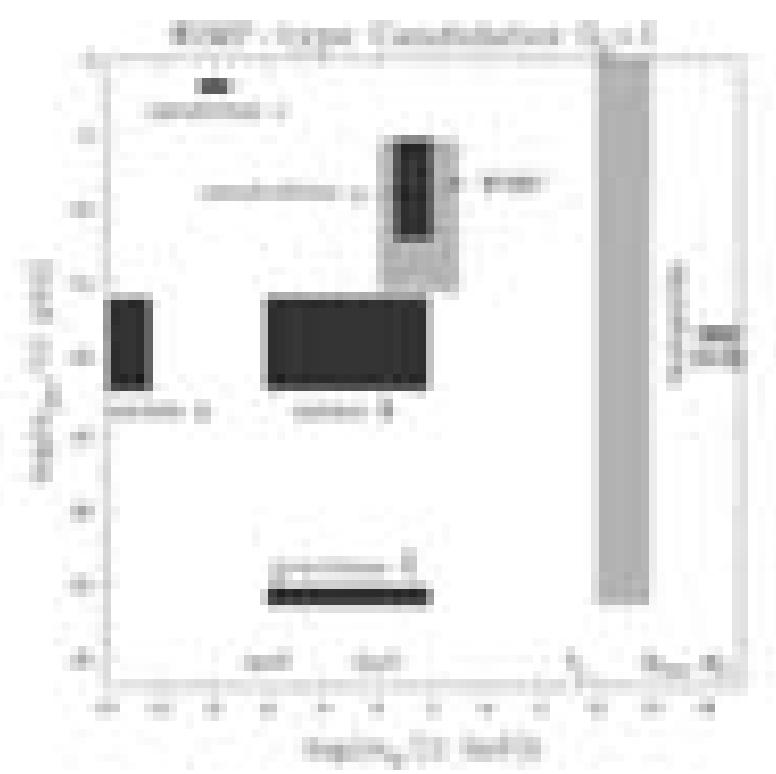

Fig. 1. Cross sections and masses of WIMP dark matter candidates. Black hole remnant (BHR) is included.

In a recent paper, ${ }^{12}$ a generalized uncertainty principle (GUP) ${ }^{13,14,15,16}$ was invoked to argue that the total collapse of a black hole may be prevented. These arguments then lead to the existence of a stable BHR at around the Planck mass and size. Such a BHR is totally inert, with no attributes other than gravitational interaction, and is thus a natural candidate for DM. It remains unclear, however, whether such a notion can be smoothly incorporated into the standard cosmology.

We note that certain inflation models naturally induce a large number of small black holes. As a specific example, in this Letter we demonstrate that the hybrid inflation ${ }^{18,19,20}$ cosmology can in principle yield the necessary abundance of primordial BHRs for them to be the primary source of dark matter. We show that such a construction is not fine-tuned.

\section{Generalized Uncertainty Principle and Black Hole Remnant}

As a result of string theory, ${ }^{14}$ or noncommutative spacetime algebra, ${ }^{15,17}$ or general considerations of quantum mechanics and gravity, ${ }^{13,16}$ the standard uncertainty principle must be modified when the gravity effect is included. A heuristic derivation may be made on dimensional grounds. Consider a particle such as an electron being observed by means of a photon with momentum $p$. The usual Heisenberg argument leads to an electron position uncertainty given by $\hbar / \Delta p$. But we should add to this a term due to the gravitational interaction between the electron and the photon, and that term must be proportional to $G$ times the photon energy, or $G p c$. Since the 
electron momentum uncertainty $\Delta p$ will be of order of $p$, we see that on dimensional grounds the extra term must be of order $G \Delta p / c^{3}$. Note that there is no $\hbar$ in the extra term when expressed in this way. The effective position uncertainty is therefore

$$
\Delta x \geq \frac{\hbar}{\Delta p}+\xi^{2} l_{p}^{2} \frac{\Delta p}{\hbar},
$$

where $l_{p}=\left(G \hbar / c^{3}\right)^{1 / 2} \approx 1.6 \times 10^{-33} \mathrm{~cm}$ is the Planck length. Here the dimensionless coefficient $\xi \sim \mathcal{O}(1)$ can be considered either as related to the string tension from the string theory's motivation, or simply as a factor to account for the imprecision of our heuristic derivation which can only be fixed by a precise theory of quantum gravity in the future. Note that Eq.(1) has a minimum value of $\Delta x_{\min }=2 \xi l_{p}$, so the Planck length (or $\xi l_{p}$ ) plays the role of a fundamental length.

The Hawking temperature for a spherically symmetric black hole may be obtained in a heuristic way with the use of the standard uncertainty principle and general properties of black holes. ${ }^{12}$ In the vicinity of the black hole surface there is an intrinsic uncertainty in the position of any vacuum-fluctuating particle of about the Schwarzschild radius, $\Delta x \approx r_{s}=2 G M_{\mathrm{BH}} / c^{2}$, due to the behavior of its field lines $^{21}$ - as well as on dimensional grounds. This leads to a momentum uncertainty $\Delta p$. Identifying $\Delta p c$ as the characteristic energy of the emitted photon, and thus as a characteristic temperature (with the insertion of a calibration factor $1 / 4 \pi$ ), one arrives at the celebrated Hawking temperature, $T_{\mathrm{H}}=\hbar c^{3} / 8 \pi G M_{\mathrm{BH}}$.

Applying the same argument, but invoking the GUP, one then finds a modified black hole temperature,

$$
T_{\mathrm{GUP}}=\frac{m_{p} c^{2}}{4 \pi} \frac{\mu}{\xi^{2}}\left[1 \mp \sqrt{1-\xi^{2} / \mu^{2}}\right],
$$

where $\mu \equiv M_{B H} / m_{p}$ is the BH mass in units of the Planck mass: $m_{p}=(\hbar c / G)^{1 / 2} \approx$ $1.2 \times 10^{19} \mathrm{GeV}$. This agrees with the Hawking result for large mass if the negative sign is chosen, whereas the positive sign has no evident physical meaning. Note that the temperature becomes complex and unphysical for mass less than $\xi m_{p}$ and Schwarzschild radius less than $2 \xi l_{p}$, the minimum size allowed by the GUP. At $\mu=\xi$, $T_{\mathrm{GUP}}$ is finite but its slope is infinite, which corresponds to zero heat capacity of the black hole. The $\mathrm{BH}$ evaporation thus comes to a stop, and what remains is a inert BHR with mass $\mu=\xi$.

If there are $g$ species of relativistic particles, then the $\mathrm{BH}$ evaporation rate (assuming the Stefan-Boltzmann law) is

$$
\dot{\mu}=-\frac{16 g}{t_{c h}} \frac{\mu^{6}}{\xi^{8}}\left[1-\sqrt{1-\xi^{2} / \mu^{2}}\right]^{4},
$$

where $t_{c h}=60(16)^{2} \pi t_{p} \approx 4.8 \times 10^{4} t_{p}$ is a characteristic time for $\mathrm{BH}$ evaporation, and $t_{p}=\left(\hbar G / c^{5}\right)^{1 / 2} \approx 0.54 \times 10^{-43} \mathrm{sec}$ is the Planck time. Note that the energy output given by Eq.(3) is finite at the end point where $\mu=\xi$, i.e., $d \mu /\left.d t\right|_{\mu=\xi}=$ $-16 g /\left(\xi^{2} t_{c h}\right)$. Thus the hole with an initial mass $\mu_{i}$ evaporates to a remnant in a 
time given by

$$
\begin{aligned}
\tau & =\frac{t_{c h}}{16 g}\left[\frac{8}{3} \mu_{i}^{3}+\frac{8}{3}\left(\mu_{i}^{2}-\xi^{2}\right)^{3 / 2}-4 \xi^{2}\left(\mu_{i}^{2}-\xi^{2}\right)^{1 / 2}\right. \\
& \left.-8 \xi^{2} \mu_{i}+4 \xi^{3} \cos ^{-1} \frac{\xi}{\mu_{i}}+\frac{19}{3} \xi^{3}-\frac{\xi^{4}}{\mu_{i}}\right] \\
\approx & \frac{\mu_{i}^{3}}{3 g} t_{c h}, \quad \mu_{i} \gg 1 .
\end{aligned}
$$

The evaporation time in the $\mu_{i} \gg 1$ limit agrees with the standard Hawking picture.

\section{Hybrid Inflation and Black Hole Production}

Next we combine this notion with the hybrid inflation proposed by A. Linde. ${ }^{18,22}$ In the hybrid inflation model two inflaton fields, $(\phi, \psi)$, are invoked. Governed by the inflation potential,

$$
V(\phi, \psi)=\left(M^{2}-\frac{\sqrt{\lambda}}{2} \psi^{2}\right)^{2}+\frac{1}{2} m^{2} \phi^{2}+\frac{1}{2} \gamma \phi^{2} \psi^{2},
$$

$\phi$ first executes a "slow-roll" down the potential, and is responsible for the more than 60 e-folds expansion while $\psi$ remains zero. When $\phi$ eventually reduces to a critical value, $\phi_{c}=\left(2 \sqrt{\lambda} M^{2} / \gamma\right)^{1 / 2}$, it triggers a phase transition that results in a "rapid-fall" of the energy density of the $\psi$ field, which lasts only for a few e-folds, that ends the inflation.

The equations of motion for the fields are

$$
\begin{aligned}
\ddot{\phi}+3 H \dot{\phi} & =-\left(m^{2}+\gamma \psi^{2}\right) \phi, \\
\ddot{\psi}+3 H \dot{\psi} & =\left(2 \sqrt{\lambda} M^{2}-\gamma \phi^{2}-\lambda \psi^{2}\right) \psi,
\end{aligned}
$$

subject to the Friedmann constraint,

$$
H^{2}=\frac{8 \pi}{3 m_{p}^{2}}\left[V(\phi, \psi)+\frac{1}{2} \dot{\phi}^{2}+\frac{1}{2} \dot{\psi}^{2}\right]
$$

The solution for the $\psi$ field in the small $\phi$ regime, measured backward from the end of inflation, is

$$
\psi(N(t))=\psi_{e} \exp (-s N(t)),
$$

where $N(t)=H_{*}\left(t_{e}-t\right)$ is the number of e-folds from $t$ to $t_{e}$ and $s=-3 / 2+(9 / 4+$ $\left.2 \sqrt{\lambda} M^{2} / H_{*}^{2}\right)^{1 / 2}$ and $H_{*} \simeq \sqrt{8 \pi / 3} M^{2} / m_{p}$.

We now show how a large number of small black holes can result from the second stage of inflation. Quantum fluctuations of $\psi$ induce variations of the starting time of the second stage inflation, i.e., $\delta t=\delta \psi / \dot{\psi}$. This translates into perturbations on the number of e-folds, $\delta N=H_{*} \delta \psi / \dot{\psi}$, and therefore the curvature contrasts.

It can be shown that ${ }^{23}$ the density contrast at the time when the curvature perturbations re-enter the horizon is related to $\delta N$ by

$$
\delta \equiv \frac{\delta \rho}{\rho}=\frac{2+2 w}{5+3 w} \delta N
$$


where $p=w \rho$ is the equation of state of the universe at reentry. From Eq.(8), it is easy to see that $\dot{\psi}=s H_{*} \psi$. At horizon crossing, $\delta \psi \sim H_{*} / 2 \pi$. So with the initial condition (at $\left.\phi=\phi_{c}\right) \psi \sim H_{*} / 2 \pi$, we find that $\delta N \sim 1 / s$. Thus

$$
\delta \sim \frac{2+2 w}{5+3 w} \frac{1}{s} .
$$

As $w$ is always of order unity, we see that the density perturbation can be sizable if $s$ is also of order unity. With an initial density contrast $\delta(m) \equiv \delta \rho /\left.\rho\right|_{m}$, the probability that a region of mass $m$ becomes a $\mathrm{PBH}$ is ${ }^{24}$

$$
P(m) \sim \delta(m) e^{-w^{2} / 2 \delta^{2}} .
$$

Let us assume that the universe had inflated $e^{N_{c}}$ times during the second stage of inflation. From the above discussion we find ${ }^{22}$

$$
e^{N_{c}} \sim\left(\frac{2 m_{p}}{s H_{*}}\right)^{1 / s} .
$$

At the end of inflation the physical scale that left the horizon during the phase transition is $H_{*}^{-1} e^{N_{c}}$. If the second stage of inflation is short, i.e., $N_{c} \sim \mathcal{O}(1)$, then the energy soon after inflation may still be dominated by the oscillations of $\psi$ with $p=0$, and the scale factor of the universe after inflation would grow as $\left(t H_{*}\right)^{2 / 3}$. The scale $\left(t H_{*}\right)^{2 / 3} H_{*}^{-1} e^{N_{c}}$ became comparable to the particle horizon $(\sim t)$, or $t \sim\left(t H_{*}\right)^{2 / 3} H_{*}^{-1} e^{N_{c}}$, when

$$
t \sim t_{h}=H_{*}^{-1} e^{3 N_{c}} .
$$

At this time if the density contrast was $\delta \sim 1$, then BHs with size $r_{s} \sim H_{*}^{-1} e^{3 N_{c}}$ would form with an initial mass

$$
\mu_{i} \simeq \frac{m_{p}}{H_{*}} e^{3 N_{c}} \sim \frac{m_{p}}{H_{*}}\left(\frac{2 m_{p}}{s H_{*}}\right)^{3 / s} .
$$

Since $H_{*}$ depends on $M$ while $s$ on $M$ and $\lambda$, the initial BH mass depends only on the mass and the coupling in the $\psi$-sector of the hybrid inflation.

\section{Black Hole Remnants as Dark Matter}

By identifying BHRs as DM and assuming hybrid inflation as the progenitor of PBHs, we in effect impose a constraint between $H_{*}$ and $s$ (or equivalently, $M$ and $\lambda$ ). Though constrained, these parameters are not so fine-tuned, as we will show in the following analysis.

We wish to estimate the present abundance of the BHRs created by hybrid inflation. To do so we should track the evolution of the post-inflation PBHs through different cosmological epochs. The newly introduced "black hole epoch" $\left(t_{h} \leq t \leq \tau\right)$ would in principle involve evaporation and mergers of PBHs as well as their accretion of radiation; the details of which can be intricate. For the purpose of a rough estimate and as a good approximation, however, one is safe to neglect these detailed dynamics and only keep track of the $\mathrm{BH}$ evaporation throughout the $\mathrm{BH}$ epoch. We assume 
that the universe was matter dominant at the time $t_{h}$ when PBHs were formed. Due to Hawking evaporation, the universe would later become radiation dominant. Since the rate of $\mathrm{BH}$ evaporation rises sharply in its late stage, the crossing time $t_{x}$ of this transition can be roughly estimated by integrating Eq.(3) from $\mu_{i}$ to $\mu_{i} / 2$. This gives $t_{x} \sim 7 / 8 \tau$. When the Hubble expansion effect is included, where the radiation density dilutes faster than the matter density by one power of the scale factor, the crossing time would be even closer to $\tau$. For our purpose, we can simply assume that the entire $\mathrm{BH}$ epoch was matter dominant.

The radiation to matter density ratio at the end of $\mathrm{BH}$ epoch, with Hubble expansion included, can then be estimated as

$$
\frac{\Omega_{\gamma, \tau}}{\Omega_{\mathrm{BHR}, \tau}} \sim \frac{1}{\xi} \int_{t_{h}}^{\tau} d t \dot{\mu}\left(\frac{t}{\tau}\right)^{2 / 3} \sim \mathcal{O}(1) \frac{\mu_{i}}{\xi} .
$$

Not surprisingly, this ratio is just roughly the initial $\mathrm{BH}$ mass over what remains in its remnant. As we will see below, the typical BH mass in our scenario, while small in astrophysical sense, is nonetheless much larger than the Planck mass, i.e., $\mu_{i} \gg 1$. Furthermore, the effective reheating temperature through Hawking evaporation in this case would be much higher than the energy scales associated with the standard model of particle physics and baryogenesis. We thus assume that the standard cosmology would resume after the black hole epoch. To conform with the standard cosmology, our assumption that DM is predominantly contributed from BHR demands that, by the time $t \sim t_{e q} \sim 10^{12}$ sec the density contributions from radiation and BHR should be about equal, namely,

$$
\frac{\Omega_{\gamma, t_{e q}}}{\Omega_{\mathrm{BHR}, t_{e q}}} \sim 1 \sim\left(\frac{\tau}{t_{e q}}\right)^{1 / 2} \frac{\Omega_{\gamma, \tau}}{\Omega_{\mathrm{BHR}, \tau}} \sim\left(\frac{\tau}{t_{e q}}\right)^{1 / 2} \frac{\mu_{i}}{\xi} .
$$

Since $\tau$ is uniquely determined by the initial BH mass $\mu_{i}$ (cf. Eq.(4)), the above condition translates into a constraint on $H_{*}$ and $s$ in hybrid inflation through Eq.(14):

$$
\frac{m_{p}}{H_{*}}\left(\frac{2 m_{p}}{s H_{*}}\right)^{3 / s} \sim\left(3 g \xi^{2} \frac{t_{e q}}{t_{c h}}\right)^{1 / 5} \text {. }
$$

Figure 2 shows the region in the $\left(H_{*}, s\right)$ parameter space that satisfies the above condition. We assume $g=100$. The width of the band reflects the uncertainty on the value of $\xi$. Here we assume $0.3 \leq \xi \leq 3.0$. We see that within the constraint that $s$ be of the order unity so that the metric perturbation $\delta$ be not exponentially small, there exists a wide range of $H_{*}$ that could produce the right amount of PBHs.

As an example, we take $H_{*} \sim 5 \times 10^{13} \mathrm{GeV}$ and $s \sim 3$. Assume that the universe was matter-dominated when the curvature perturbation reentered the horizon. Then the density contrast is $\delta \sim 1 / 7$, and the fraction of matter in the BH is $P(m) \sim 10^{-2}$. From Eq.(12), $e^{N_{c}} \sim 54$. So the total number of e-folds is $N_{c} \sim 4$. The black holes were produced at the moment $t_{h} \sim 2 \times 10^{-33}$ sec, and had a typical mass $M_{\mathrm{BH} i} \sim 4 \times 10^{10} m_{p}$. Let $g \sim 100$. Then the time it took for the BHs to reduce to remnants, according to Eq.(4), is $\tau \sim 5 \times 10^{-10}$ sec. The "black hole epoch" thus ended in time before baryogenesis and other subsequent epochs in the standard 


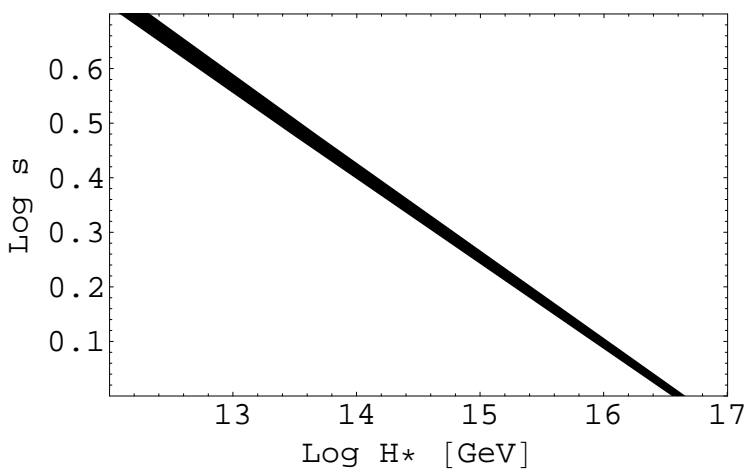

Fig. 2. Region in the hybrid inflation $\left(H_{*}, s\right)$ parameter space where the induced black hole remnants would provide the right abundance for dark matter.

cosmology. As suggested in Ref. 22, such a post-inflation PBH evaporation provides an interesting mechanism for reheating. Note that due to the continuous evaporation process and the Hubble expansion, the $\mathrm{BH}$ reheating should result in an effective temperature which is sufficiently lower than the Planck scale.

\section{Discussion}

Our arguments for the existence of BHR based on GUP is heuristic. The search for its deeper theoretical foundation is currently underway. As interactions with BHR are purely gravitational, the cross section is extremely small, and direct observation of BHR seems unlikely. One possible indirect signature may be associated with the cosmic gravitational wave background. Unlike photons, the gravitons radiated during evaporation would be instantly frozen. Since, according to our notion, the $\mathrm{BH}$ evaporation would terminate when it reduces to a BHR, the graviton spectrum should have a cutoff at Planck mass. Such a cutoff would have by now been redshifted to $\sim \mathcal{O}\left(10^{4}\right) \mathrm{GeV}$. Another possible GW-related signature may be the GWs released during the gravitational collapse at $t \sim t_{h}$. The frequencies of such GWs would by now be in the range of $\sim 10^{7}-10^{8} \mathrm{~Hz}$. It would be interesting to investigate whether these signals are in principle observable. Another possible signature may be some imprints on the CMB fluctuations due to the thermodynamics of PBH-CMB interactions. These will be further investigated.

\section{Acknowledgments}

I deeply appreciate my early collaborations and fruitful discussions with Ronald J. Adler. I also thank S. Dimopoulos, A. Green, A. Linde, M. Rees, M. Shmakova, and J. Silk for helpful discussions. This work is supported by the Department of Energy under Contract No. DE-AC03-76SF00515. 


\section{References}

1. P. Gondolo, astro-ph/0403064.

2. L. Roszkowski, "An Overview of WIMP-Like Dark Matter Candidate and the Prospect of Neutralino Detection", talk given at 6th UCLA Symposium on Sources and Detection of Dark Matter and Dark Energy in the Universe, Marina del Ray, California, Feb. 1820, 2004.

3. Ya. B. Zeldovich and I. D. Novikov, Sov. Astron. 10, 602 (1966).

4. S. W. Hawking, Mon. Not. R. Astron. Soc. 152, 75 (1971).

5. J. H. MacGibbon, Nature 329, 308 (1987); J. D. Barrow, E. J. Copeland, A. R. Liddle, Phys. Rev. D 46, 645 (1992).

6. B. J. Carr, J. H. Gilbert, and J. E. Lidsey, Phys. Rev. D 50, 4853 (1994); J. E. Lidsey, B. J. Carr, and J. H. Gilbert, arXiv: astro-ph/9406028.

7. D. N. Spergel et al., Astrophys. J. Suppl. 148175 (2003).

8. See, for example, M. Yu. Khlopov, Cosmoparticle Physics, (World Scientific, 1999).

9. J. D. Bekenstein, Phys. Rev. D 7, 2333 (1973); J. D. Bekenstein, Lettere al Nuovo Cimento 4, 737 (1972).

10. S. W. Hawking, Comm. Math. Phys. 43, 199 (1974).

11. M. K. Parikh and F. Wilczek, Phys. Rev. Lett. 85, 5042 (2000), and references therein.

12. R. J. Adler, P. Chen, and D. Santiago, Gen. Rel. Grav. 33 (12), 2101 (2001); arXiv: gr-qc/0106080.

13. C. A. Mead, Phys. Rev. 135, B849 (1964); Phys. Rev. 143990 (1966).

14. G. Veneziano, Europhys. Lett. 2, 199 (1986); E. Witten, Phys. Today, Apr. 24 (1996).

15. M. Maggiore, Phys. Lett. B304, 65 (1993); F. Scardigli, Phys. Lett. B452, 39 (1999).

16. R. J. Adler and D. I. Santiago, Mod. Phys. Lett. A14, 1371 (1999).

17. There is, however, a different form of GUP based on the noncommutative spacetime algebra where our form (Eq.(1)) is a special case when $\Delta x \sim \Delta t$. See, for example, T. Yoneya, Prog. Theor. Phys. 103, 1081 (2000); R. Brandenberger and P.-M. Ho, Assoc. Asian Pac. Phys. Soc. Bull. 12, 10 (2002).

18. A. Linde, Phys. Lett. B259, 38 (1991); Phys. Rev. D 49, 748 (1994).

19. E. J. Copeland, A. R. Liddle, D. H. Lyth, E. D. Stewart, and D. Wands, Phys. Rev. D 49, 6410 (1994).

20. For a recent review, see D. H. Lyth and A. Riotto, Phys. Rep. 314, 1 (1999); A. Linde, Phys. Rep. 333-334, 575 (2000).

21. R. S. Hanni and R. Ruffini, in Black Holes, eds. C. DeWitt and B. S. DeWitt, R57 (Gordon and Breach, 1973); R. J. Adler and T. K. Das, Phys. Rev. D 14, 2474 (1976).

22. J. Garcia-Bellido, A. Linde, and D. Wands, Phys. Rev. D 54, 6040 (1996).

23. A. R. Liddle and D. H. Lyth, Phys. Rep. 231, 1 (1993).

24. B. J. Carr and S. W. Hawking, Mon. Not. R. Astron. Soc. 168, 399 (1974); B. J. Carr, Astrophys. J. 201, 1 (1975). 